\title{
RESTLESS LEGS SYNDROME ASSOCIATED WITH CARDIAC FAILURE AND AGGRAVATED AFTER VALVULAR REPLACEMENT
}

\section{Vesper's curse?}

\author{
Raimundo Nonato Delgado Rodrigues', Aída Alexandra Alvim de Abreu e Silva Rodrigues², \\ Jeana Torres Corso ${ }^{3}$, Thaisa Fabiana Peixoto ${ }^{3}$
}

The restless legs syndrome (RLS) is a neurological disorder characterized by symptoms of discomfort (usually paresthesias) in the limbs, predominating in the afternoon, present mainly at rest and alleviated by movement. The symptoms usually appear in the late afternoon or at night, which may bring on a significant increase in sleep latency and contribute to poor sleep quality'. Since the prevalence of RLS increases with age ${ }^{2}$, heterogeneous conditions are likely to be identified in association with other illnesses typical of advanced age, making diagnoses more complex, and indicating the importance of studying these secondary forms, and their possible physiopathological mechanisms.

We report a case of RLS associated to important abnormalities of the cardiac function in an elderly patient, who underwent several cardiac surgical interventions, and discuss the possible implications of this cardiac dysfunction in the etiology and maintenance of the motor symptoms. This case is reported after approval of our Institution and informed patient's consent.

\section{CASE}

A 73-year-old female, with congestive cardiac failure and severe mitral valvulopathy presented a history of chronic insomnia. She had been submitted to three valve replacement surgeries over a period of 30 years. After the last surgery (January 2007) she developed a temporary renal failure. She also reported diabetes mellitus, hypothyroidism, osteoporosis and, in childhood, rheumatic fever. She had been prescribed opioids during the immediate post-operatory period. The patient came to the visit on 15 February 2007 taking several medications including pramipexole (used for less than thirty days), clonaze- pam, codein+paracetamol, diazepam and iron quelate-glicinate (prescribed less than 2 months prior). She had also taken dolantin one week before the visit for "pains in the legs".

One year before the patient began to report a feeling of "impatience", "agony" and "restlessness" in her legs, being forced to move around to provide some relief. These symptoms were predominantly nocturnal, and hampered the onset of sleep. She reported similar symptoms in her upper limbs, though less intense. Nearly one month before her neurological check-up, and immediately after her last cardiac surgery, the patient noticed a significant worsening of lower limb discomfort.

The physical examination revealed a depressed mood with no important neurological alterations.

A polysomnogram taken some days after the first visit observed: increase in sleep latency (100 minutes), superficial sleep (stages $1+2=84 \%$ ) with low efficiency $(37 \%)$ rates, a percentual decrease in REM sleep (0.5\%), and a micro arousal rate of six per hour. Eleven respiratory events per hour of sleep (ranging from apnea to hypopnea) were noticed. Oxyhemoglobin saturation varied between $90 \%$ and $78 \%$ during the night. Thirteen periodic limb movements were detected per hour of sleep.

An analysis of iron metabolism revealed serum ferritin levels of $182 \mathrm{ng} / \mathrm{mL}$ (normal 10-280 ng/mL).

In the weeks following the first visit the patient's condition deteriorated, in spite of the re-adjustment of pramipexole and introduction of trazodone $100 \mathrm{mg}$ at night. She reported discomfort throughout her body needing to move around during most of the night, thus not being able to sleep. At that occasion, oxycodone $10 \mathrm{mg}$ and subsequently $20 \mathrm{mg}$ was prescribed for the evening. After that, improvements were noticed in both the sensitive-motor symptoms and sleep quality.

Her most recent echocardiogram $(\operatorname{Jan} 3,2008)$ revealed an

SÍNDROME DAS PERNAS INQUIETAS ASSOCIADA A INSUFICIÊNCIA CARDÍACA E AGRAVADA APÓS TROCA VALVULAR: MALDIÇÃO DE VÉSPER?

'MD, MSc, PhD, Neurologist, Sleep Disorders Center, Hospital Universitário de Brasília, University of Brasília, Brasília DF, Brazil; ${ }^{2} \mathrm{MD}$, PulmonologistSleep Disorders Center, Hospital Universitário de Brasília, University of Brasília, Brasília DF, Brazil; ${ }^{3}$ Medicine Student, University of Brasília, Campus Darcy Ribeiro s/n, Brasilia DF, Brazil.

Received 14 March 2008, received in final form 5 May 2008. Accepted 29 May 2008.

Dr. Raimundo Nonato Delgado Rodrigues - SQN 206 K 303 - 70844-010 Brasilia DF - Brasil.E-mail: reno@unb.br 
ejection fraction of $47 \%$, a slight increase in the left atrium, a moderate increase in the right-side cavities, a small systolic deficit of the left and right ventricles, an important tricuspid insufficiency and pulmonary arterial hypertension. A normofunctioning prosthetic biologic mitral valve was observed.

At present the patient remains neurologically stable, although she abandoned the treatment with dopaminergic agonist keeping a daily use of oxycodone $20 \mathrm{mg}$ at night.

\section{DISCUSSION}

The pathophysiology of RLS has yet to be fully elucidated. Besides the loss of inhibition of subcortical movement pattern generators ${ }^{3}$, there probably exists a vesperal increase in the excitability of the spinal motoneurons, reinforcing the final portion of the spinal reflex ${ }^{4}$. The literature has been interested in the prevalence of RLS under special circumstances, such as during renal insufficiency and pregnancy ${ }^{1,3,5}$. More recently, however, special consideration has been given to post-operatory patients, especially those who have undergone major thoracic surgery ${ }^{6,7}$.

Cortese et al. ${ }^{7}$ described a case of pediatric RLS whose symptoms began after heart surgery to correct a slight atrial malformation. The patient did not report any prior complaint suggesting RLS, although many cases existed in the family. The symptoms of RLS persisted after surgery. This is a curious observation, especially considering that Högl et al. ${ }^{8}$ have described only temporary RLS symptoms in $8.7 \%$ of a series of 161 patients submitted to raquianesthesia.

The presence of RLS after major thoracic surgery (cardiac or pulmonary) was also studied by other researchers. The available data suggest a prevalence rate between $20 \%{ }^{9}$ and $47.6 \%{ }^{6}$, with possible higher risk among females and in the presence of thyroid disorders ${ }^{6}$.

However, it seems that most of the few existing studies were cross-sectional and did not conduct causal analyses nor tried to determine the prior existence of RLS in a specific population. Such data would be most interesting to obtain as some studies have indicated that, in certain situations, heart or kidney transplants may have an important and positive influence on the clinical course of RLS symptoms ${ }^{10,11}$.

In the patient described in this study, several risk factors associated with the development of RLS were present, namely diabetes mellitus, hypothyroidism, as well as an episode of post-operatory renal failure. The patient was also a female and elderly.

The reasons for the appearance or aggravation of RLS symptoms in patients who have undergone heart surgery are not clear. Cerebral iron deficiency secondary to the inevitable blood loss during this type of surgical proce- dure, or immediate post-operatory metabolic alterations (hypocalcemia, hypomagnesemia) may also contribute ${ }^{6,7}$. Prolonged immobilization and sleep deprivation may be pointed out as additional aggravating factors ${ }^{6-8}$. The patient in question presented normal serum ferritin levels. Nevertheless, as is known, this condition may coexist with deficient brain levels, which are ultimately responsible for the sensitive-motor disorder ${ }^{12}$. On the other hand, we cannot formally dismiss the influence of an unfavorable hemodynamic condition that implicitly accompanies patients requiring this type of surgery.

It is known that the reduction of cardiopulmonary complacency in certain situations may be responsible for pain and paresthesias in the lower limbs during the afternoon, sometimes associated with cramps and fasciculations, which is known as "Vesper's curse"13. From a practical perspective, an increase in the right atrium filling pressure secondary to a state of chronic heart failure causes a delay in venous circulation that may lead to an increase in the pressure and enlargement of the lumbar veins with a relative narrowing of the spinal canal and reduced oxygenation ${ }^{1,13}$. It is possible to imagine that, in this condition, there may exist an increase in afferent sensorial stimuli which, associated to the spinal hyperexcitability present in patients with RLS, may act as an important generating factor for the motor symptoms.

"Vesper's curse" has been considered an uncommon occurrence ${ }^{1}$. However, a more detailed analysis of the literature demonstrates that this condition has already been described in several situations, including those associated with spondylothic alterations or lumbar spondylolisthesis ${ }^{13}$.

Hanly et al. reported a case of a patient with congestive heart failure, chronic insomnia and an important periodic limb movements (PLM) syndrome, whose condition improved dramatically three months after heart transplantation ${ }^{10}$. The authors, even if they did not explicitly refer to "Vesper's curse", considered that a low cardiac output could be in part responsible for the presence of PLM and stated that the correction of the ventricular dysfunction contributed to the overall improvement of the sleep disruption.

The patient described in this paper presented a highly unfavorable cardiac and hemodynamic condition, probably secondary to a chronic rheumatic valvular cardiopathy. In spite of the three prior valvular replacements, a recent echocardiographic evaluation demonstrated significant alterations represented by a low ejection fraction (47\%), signs of hypertrophy in the right chambers and pulmonary hypertension. Its seems possible that the patho physiological process, which goes by the inauspicious 
name of "Vesper's curse", has fully developed, and contributed significantly towards the progressive aggravation of the symptoms of RLS, in spite of the many attempts to surgically correct the valvulopathy.

Furthermore, a recent study of patients with myocardiopathy using imaging, electromyographic and evoked potential techniques demonstrated the existence of a significant link between pulmonary hypertension and the presence of symptomatic lumbar spine stenosis during the afternoon ${ }^{14}$.

On the other hand, the appearance of RLS symptoms after major thoracic surgery may be relatively delayed, which certainly hampers the precise determining of its prevalence. This is generally attributed to the early use of opiate analgesics to control post-operatory pain ${ }^{7,8}$, as was the case for this patient.

In conclusion, the case reported here and the review of the pertinent literature suggest that the so-called "Vesper's curse" may not be so uncommon and may represent a significant cause of secondary RLS or even contribute towards its aggravation in susceptible patients with associated cardiovascular disease. Cardiac surgery per se appears to be a causative factor of only minor importance to RLS. Contrariwise, it may contribute positively to the resolution of motor symptoms through the improvement of cardiac function and hemodynamic recovery.

\section{REFERENCES}

1. Garcia-Borreguero D, Odin P, Schwarz C. Restless legs syndrome: an overview of the current understanding and management. Acta Neurol Scand 2004;109:303-317.

2. Síndrome das pernas inquietas: diagnóstico e tratamento. Opinião de especialistas brasileiros. Arq Neuropsiquiatr 2007;65:721-727.

3. Allen RP. Controversies and challenges in defining the etiology and pathophysiology of restless legs syndrome. Am J Med 2007;120(Suppl):S13-S21.

4. Bara-Jimenez W, Aksu M, Graham B, Sato S, Hallett M. Periodic limb movements in sleep: state-dependent excitability of the spinal flexor reflex. Neurology 2000;54:1609-1616.

5. Goffredo GS Filho, Gurimi CC, Purysko AS, Silva HC, Elias IEF. Restless legs syndrome in patients on chronic hemodialysis in a Brazilian city: frequency, biochemical findings and comorbidities. Arq Neuropsiquiatr 2003;61:723-727.

6. Minai OA, Golish JA, Yataco JC, Budev MM, Blazey H, Giannini RN Restless legs syndrome in lung transplant recipients. J Heart Lung Transplant 2007;26:24-28.

7. Cortese S, Konofal E, Lecendreux M, Mouren MC. Restless legs syndrome triggered by heart surgery. Pediatr Neurol 2006;35:223-226.

8. Högl B, Frauscher B, Seppi K, Ulmer H, Poewe W. Transient restless legs syndrome after spinal anesthesia: a prospective study. Neurology 2002;59:1705-1707.

9. Javaheri S, Abraham WT, Brown C, Nishiyama H, Giesting R, Wagoner LE Prevalence of obstructive sleep apnoea and periodic limb movement in 45 subjects with heart transplantation. Eur Heart J 2004;25:260-266.

10. Hanly P, Zuberi N. Periodic leg movements during sleep before and after heart transplantation. Sleep 1992;15:489-492.

11. Winkelmann J, Stautner A, Samtleben W, Trenkwalder C. Long-term course of restless legs syndrome in dialysis patients after kidney transplantation. Mov Disord 2002;17:1072-1076.

12. Earley CJ, Connor JR, Beard JL, Malecki EA, Epstein DK, Allen RP. Abnormalities in CSF concentrations of ferritin and transferrin in restless legs syndrome. Neurology 2000;54:1698-1700.

13. LaBan MM, Viola SL, Femminineo AF, Taylor RS. Restless legs syndrome associated with diminished cardiopulmonary compliance and lumbar spinal stenosis: a motor concomitant of "Vesper's curse". Arch Phys Med Rehabil 1990;71:384-388.

14. LaBan MM, Grabowski SR, Taylor RS. Nocturnal lumbosacral pain as a consequence of cardiomyopathy and lumbar spinal stenosis: is pulmonary hypertension the dynamic link in precipitating these symptoms of "Vesper's curse". Arch Phys Med Rehab 2003;84:E18-E19. 